\title{
Mechanisms of Cytokine-Induced Glioma Immunosuppression
}

\author{
Alexander Ksendzovsky ${ }^{*},{ }^{1}$, Roberta P. Glick ${ }^{2}$, Paul Polak ${ }^{3}$, Maria-Vittoria Simonini ${ }^{3}$, Anothony J. \\ Sharp $^{3}$, Tyler Newman ${ }^{3}$ Edward P. Cohen ${ }^{4}$ and Douglas L. Feinstein ${ }^{3}$
}

\author{
${ }^{I}$ Chicago Medical School, North Chicago, IL, USA ${ }^{2}$ Mt. Sinai Hospital Dept. Neurosurgery, Rush College of Medicine \\ Dept. Neurosurgery, University of Illinois - Chicago Dept. Anatomy and Cell Biology, Chicago, IL, USA; ${ }^{3}$ University of \\ Illinois - Chicago Dept. Anesthesia, Jesse Brown Veterans Affair Hospital, Chicago, IL, USA, ${ }^{4}$ UIC Dept. Microbiology \\ and Immunology, Chicago, IL, USA
}

\begin{abstract}
Glioma immunosuppression includes the secretion of cytokines that down-regulate the host immune response resulting in tumor survival. The mechanisms of cytokine-induced immunosuppression are not well understood and are considered in this study. Glioma cells were incubated with supernatant from activated and naïve T-cells. A separate culture of T-cells (naïve, CD3-activated, and CD3/CD28 activated) was then incubated with conditioned media from the treated glioma cells as well as individual and combination recombinant cytokines. These T-cells were tested for viability, proliferation and IFN- $\gamma$ release. Several conclusions were drawn from these experiments: cytotoxicity is not a means of glioma immunosuppression, glioma conditioned media decreases proliferation of CD3/CD28 activated T-cells acting potentially through IL10 and IGFBP, and these cytokines also decrease IFN- $\gamma$ secretion from all varieties of T-cells suggesting that T-cell differentiation away from TH1 is another potential means of immunosuppression. These results necessitate further analysis of proliferation and differentiation as potential mechanisms of immunosuppression and the incorporation of this knowledge into the production of a more efficacious tumor vaccine.
\end{abstract}

Key Words: Glioma, immunotherapy, cytokine, TGF-beta, immunosuppression.

\section{INTRODUCTION}

Gliomas have evolved several mechanisms to evade host immunity leading to enhanced tumor proliferation and limited immunotherapy effectiveness. For the last two decades, several immunosuppressive mechanisms have been explored including T-regulatory cells, immunosuppressive cytokines, antigen down-regulation, and immune-inhibitory molecules [1-3], yet no definitive conclusions have been reached. IL10 , TGF- $\beta$, PGE, and other cytokines have recently been implicated in direct immune-suppression [4-8]. Research has shed some light on cytokines' mechanisms of immunosuppression including the following: TGF- $\beta 2$ has been found to inhibit cytotoxic responses of T cells, IL-10 down- regulates MHC Class II expression and suppresses $\mathrm{T}$ cell proliferation [5-7], PGE2 was shown to regulate the initiation of an immune response, and VEGF inhibits the maturation of dendritic cells (DCs) and down-regulates MHC class II molecules $[4,8]$. Despite these promising results there is still much left to be discovered regarding mechanisms of cytokine-induced immunosuppression and this is the topic of our study.

In the present study we explored the potential mechanisms of immunosuppression from a collective sample of cytokines secreted by mouse glioma cells in response to activated immunity. Activated immunity was simulated by incubating glioma cells with conditioned media from T-cells

"Address correspondence to this author at the Chicago Medical School, North Chicago, IL, USA; Tel: (404)550-5694; Fax: (312) 996-9680; E-mail: aksendz@my.rfums.org activated with anti-CD3/CD28 antibodies. The glioma supernatant, containing cytokines secreted by glioma in response to T-cell CM, was then removed and incubated with separate T-cells. Three variants of T-cells including naïve Tcells, CD3 activated T-cells, and CD3/CD28 activated Tcells were compared and tested for cytotoxic effects, proliferation and IFN- $\gamma$ release (as a measure for potential differentiation). We also performed the above tests on individual and combinations of recombinant cytokines incubated with T-cells including IL-10, TGF- $\beta 1$, IGFBP.

\section{MATERIALS AND METHODOLOGY}

\section{Cell Lines and Experimental Animals}

The GL261 cell line, a glial tumor cell line of C57B16 mouse origin, was obtained from the National Cancer Institute, Frederick Cancer Research and Development Center. The cells were passaged in vitro in Dulbecco modified Eagle medium (Mediatech) containing $25 \mathrm{mM}$ glucose and supplemented with $10 \%$ fetal bovine serum (Gibco) and antibiotics. The medium was changed every 3 days.

\section{T-cell Isolation and Activation}

For $\mathrm{T}$ cell isolation, 2 spleens were isolated from adult C57B16 mice. The spleens were manually homogenized in PBS and the morcellate was centrifuged at $1000 \mathrm{rpm}$ for 5 minutes to obtain cells. The supernatant was discarded and the pellet was resuspended in $3 \mathrm{~mL}$ of red cell lysis buffer at room temperature for 5 minutes. Additional $7 \mathrm{~mL}$ PBS was added and the cells were again centrifuged at 1000 RPM for 5 minutes to obtain leukocytes in pellet. The pellet was resuspended in serum free DMEM to $10 \mathrm{~mL}$, counted, and 


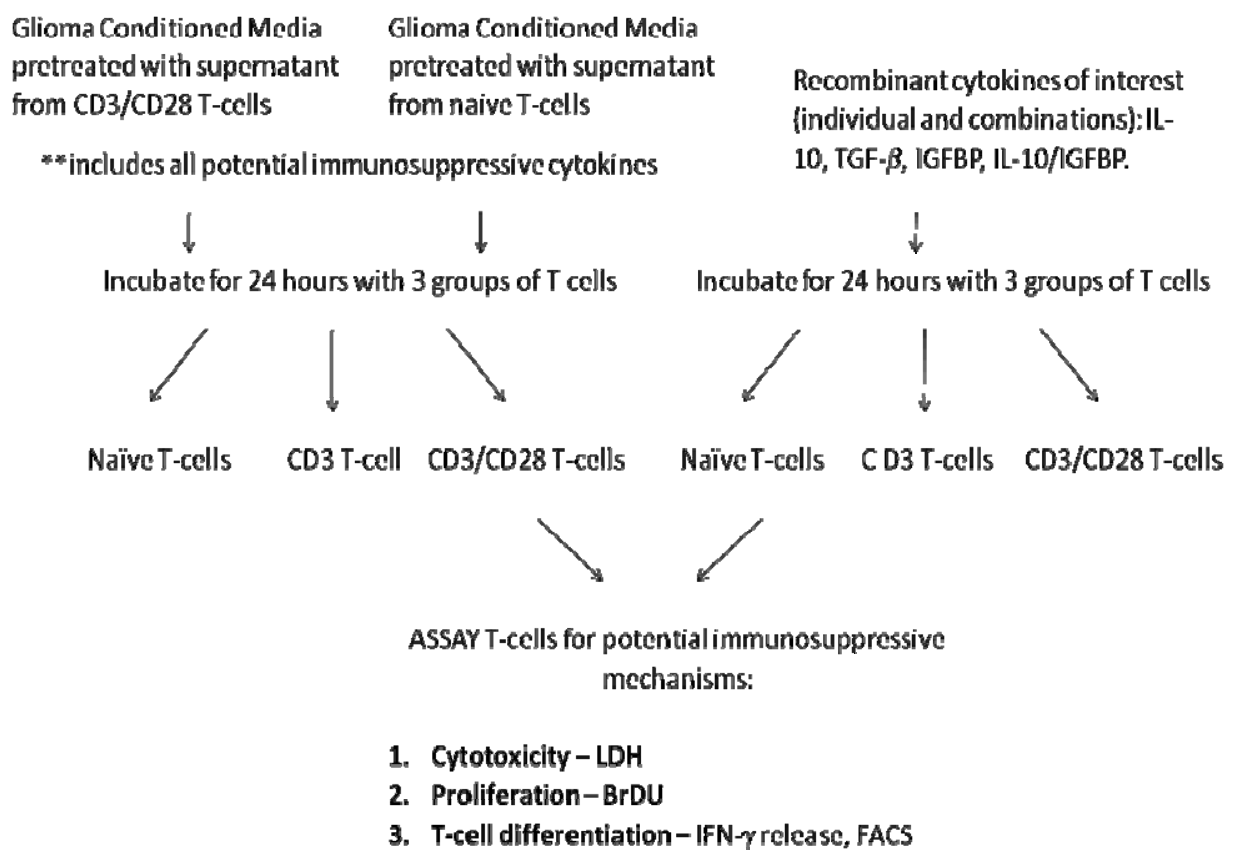

Fig. (1). Flow-chart representation of methods used in this study.

plated at a final density of $2 \mathrm{E} 6$ cells $/ \mathrm{mL}$ in a 24 well plate. The T-cells were then incubated with anti-CD3 and antiCD28 antibody for 24 hours in the following concentrations: $0.2 \mathrm{ug} / \mathrm{mL}$ and $0.5 \mathrm{ug} / \mathrm{mL}$. Supernatant from T cells activated by anti-CD3/CD28 was used as activated TCM. Supernatant from naïve T-cells was collected and used as naïve TCM. The TCM (active and naïve) was used to treat glioma cells for $24 \mathrm{hr}$. Supernatant was then collected from the glioma cells and was used to treat a separate group of T-cells in culture. The T-cells were divided into three groups: naïve, CD3treated, and CD3/CD28 treated cells and each group was treated with supernatant from glioma cells treated with either CD3/28 activated T-cell media or naïve T-cell media. After $24 \mathrm{hrs}$ of treatment, these cells were then tested for LDH release (cytotoxicity), for proliferation via a BrdU colorimetric assay, and IFN- $\gamma$ production (see Fig. 1 for flowchart of study).

\section{LDH Cytotoxicity Assay}

T-cells were treated with either supernatant from glioma cells (GCM) that had been treated with either activated or inactivated T-cell CM at either $5 \%$ or $20 \%$ final dilution, or with recombinant TGF- $\beta 1$, IL-10, IGFBP, and IL-10/IGFBP at the following concentrations: 5, 10, and $20 \mathrm{ng} / \mathrm{mL}$. After $24 \mathrm{hr}$, aliquots of the T-cell media were assayed for LDH release according to the recommended protocol from Promega's CytoTox 96 Non-Radioactive Cytotoxicity assay. In brief, the plates were spun at 500 RPM and $50 \mathrm{uL}$ of supernatant was taken from each well and placed into a 96-well plate in triplicate. Substrate was then added and absorbance was measured at $492 \mathrm{~nm}$.

\section{BrdU Proliferation Assay}

T-cell proliferation was measured using a BrdU proliferation assay on the previously mentioned T-cell groups comparing nGCM to aGCM as well as recombinant IL10/IGFBP. The GCM treated T-cells were compared to a control of T-cells treated with $1 \%$ FBS while the recombinant cytokine treated cells were compared to untreated $\mathrm{T}$ cells. These cells were plated in 96-well plates and the recommended protocol of the Colorimetric BrdU Proliferation assay from Roche was applied. Briefly, after treatments with GCM or recombinant cytokines for $24 \mathrm{hr}$, BrdU was added and cells allowed to grow for a further $24 \mathrm{hr}$. The cells were then dried and fixed to the bottom of the well, treated with anti-BrdU antibody conjugated with horseradish peroxidase, and then treated with substrate. Absorbance was then measured at $450 \mathrm{~nm}$ with a reference wavelength of $620 \mathrm{~nm}$. The BrdU proliferation assay was performed in quadruplicate.

\section{T-cell IFN- $\gamma$ secretion - ELISA}

After indicated treatments, aliquots of the T-cell media were assayed for levels of IFN- $\gamma$ by ELISA following recommended procedures (Quantikine M, R\&D Systems, Minneapolis, MN). Each sample was assayed at least in duplicate, calculation of $\mathrm{ng} / \mathrm{mL}$ cytokine determined from standard curves, and the differential secretion from T-cells from all conditions was determined.

\section{Statistical Analysis}

The Student's t-test was used to determine the statistical differences between LDH measurements and IFN- $\gamma$ secretion. ANOVA analysis was performed on BrdU results. Significance was taken at $\mathrm{P}$ values of $<0.05$.

\section{RESULTS}

\section{GCM Does Not Increase T-cell Cytotoxicity}

We first tested if treating T-cells with $\mathrm{CM}$ from activated glioma cells (aGCM, the conditioned media from glioma cells that had been activated with supernatant from activated T-cells) or from non-activated CM (nGCM, the conditioned media from glioma cells that had been treated with supernatant from naïve $\mathrm{T}$-cells) caused $\mathrm{T}$-cell damage. At the 


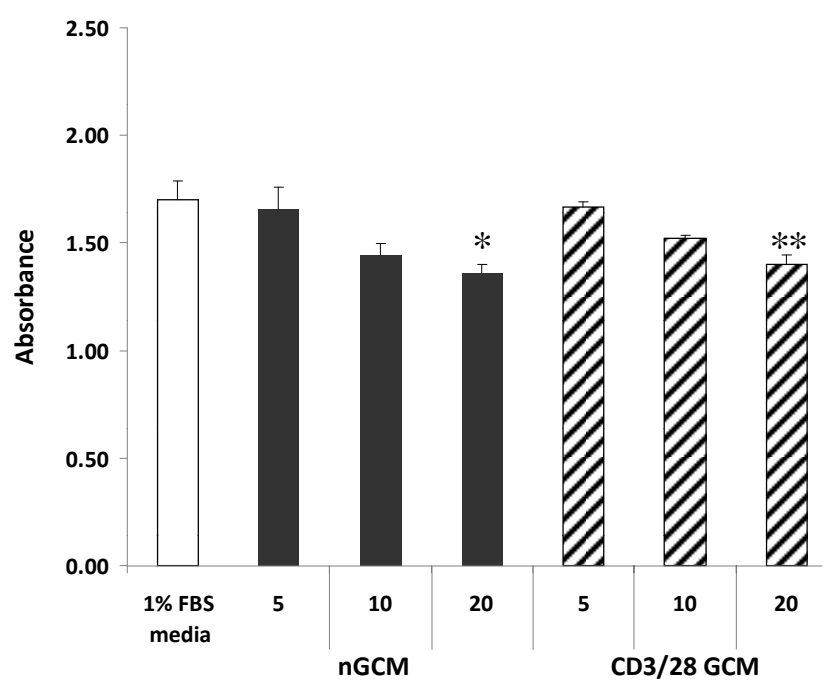

Fig. (2). Effects of GCM on T-cell activation:

T-cells were activated with antibodies to CD3 and CD28, together with naïve GCM (nGCM) or activated CM (aGCM) from glioma cells that has been incubated with $\mathrm{CM}$ from $\mathrm{CD} 3 / \mathrm{CD} 28$ treated Tcells. After $24 \mathrm{hr}$ proliferation was measured. Proliferation of CD3/CD28 activated T-cells is significantly less than controls and decreased by both nGCM and aGCM as the concentration of GCM increases. Data shown are representative of two independent experiments each done in quadruplicate. Values are means \pm standard errors. There was a statistically significant effect of nGCM $(\mathrm{P}<$ $0.05)$ and of $\mathrm{CD} 3 / \mathrm{CD} 28 \mathrm{GCM}(\mathrm{P}<0.01)$ on proliferation (1 way ANOVA). *, $\mathrm{P}<.05$; **, $\mathrm{P}<.005$ versus 1\% FBS (Bonferonni multiple comparisons).

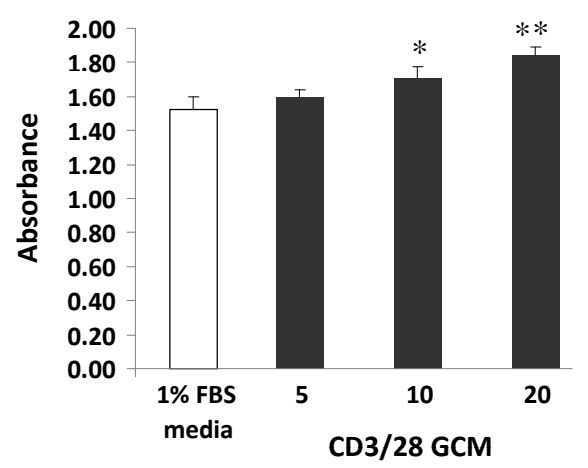

Fig (3). Effects of GCM on naive T-cells. Naïve T-cells were incubated with aGCM. After $24 \mathrm{hr}$ proliferation was measured. Activated GCM increased the proliferation of naïve T-cells as the concentration of aGCM increased. Data shown are representative of two independent experiments each done in quadruplicate. Values are means \pm standard errors. There was a statistically significant effect of CD3/CD28 GCM $(\mathrm{P}<0.005)$ on proliferation (1 way ANOVA). *, $\mathrm{P}<.05$; **, $\mathrm{P}<.005$ versus $1 \%$ FBS (Bonferonni multiple comparisons).

same time we also tested if direct treatment of T-cells with recombinant cytokines implicated in immune tolerance (IL10 , TGF- $\beta 1$, and IGFBP) would induce any death or damage. In addition, these studies were done under conditions of T-cell activation (e.g in the presence of antibodies to CD3 or to CD3 and CD28 together), or no-activation. As the concentration of GCM increased from $5 \%$ to $20 \%$, there was no increase in cytotoxicity noted in the individual T-cell classi- fications (non-activated, CD3 activated and CD3/CD28 activated). There was also no change in cytotoxicity noted among the different concentrations of the individual cytokines (IL-10, TGF- $\beta 1$, and IGFBP). Moreover, when normalized to LDH in untreated GCM and T-cell media, the LDH secretion in the treated groups was negligible. This data suggests that cytotoxocity is not an immunosuppressive mechanism involved in cytokine-mediated immunosuppression.

\section{Proliferation is Suppressed by GCM}

We then tested if any of the above treatment conditions reduced $\mathrm{T}$-cell proliferation. Results showed that the proliferation of CD3/CD28 activated T-cells was markedly decreased by both nGCM and aGCM in a dose dependent manner with greater suppression at higher concentrations of the GCM (Fig. 2). This suggests that cell-cycle arrest and a decrease in proliferation are a potential mechanism of glioma cytokine-induced immunosuppression. Interestingly, aGCM seemed to increase proliferation of naïve T-cells as the concentration of aGCM increased which was different from the response seen from CD3/CD28 activated T-cells (Fig. 3).

T-cell proliferation was not effected by incubation with any of the individual recombinant cytokines (IL-10, TGF- $\beta$, IGFBP) when used alone. However, CD3-activated T-cells co-incubated with a combination of IL-10 and IGFBP showed a significant decrease in proliferation as the concentration of IL-10/IGFBP increased from $5 \mathrm{ng} / \mathrm{mL}$ to $20 \mathrm{ng} / \mathrm{mL}$ (Fig. 4). Naïve T-cells showed a similar dose-response curve, but this data was not statistically significant (Fig. 4). While the CD3/CD28-activated T-cells co-incubated with a combination of IL-10 and IGFBP also had a decrease in proliferation as the concentration of IL10/IGFBP increased from $5 \mathrm{ng} / \mathrm{mL}$ to $10 \mathrm{ng} / \mathrm{mL}$, the proliferation was then increased as the concentration of IL10/IGFBP increased to $20 \mathrm{ng} / \mathrm{mL}$ (Fig. 5). Together, this data suggests that IL-10 and IGFBP are potentially cytokines that work together to decrease the proliferation of T-cells and that cytokines must act together to effectively cause immunosuppression in T-cells or if they do work individually, it is through a mechanism separate from proliferation.

\section{T-cell IFN- $\gamma$ Secretion is Decreased by GCM}

The supernatants from CD3/CD28 activated T-cells treated with either nGCM, aGCM, or 1\%GCM (conditioned media from glioma cells incubated with 1\% FBS) were tested for IFN- $\gamma$ release. We observed a significant decrease in IFN- $\gamma$ release from the CD3/CD28 T-cells following treatment with all forms of GCM, which was significantly lower than what was found in control T-cell IFN- $\gamma$ release (Fig. 6). This suggests that glioma-secreted cytokines modify polarity of T-cells away from IFN- $\gamma$-secreting Th1 cells, thus reducing effective T-cell mediated immunity. Because all forms of GCM had the same effect on IFN- $\gamma$ release, this response may be a base-line effect which is not affected by the immune properties of the T-cells.

\section{DISCUSSION}

Results from the present study help to elucidate several aspects of glioma-cytokine mediated immunosuppressive mechanisms. First, we have demonstrated that glioma released cytokines do not induce frank cytotoxicity in T-cells 


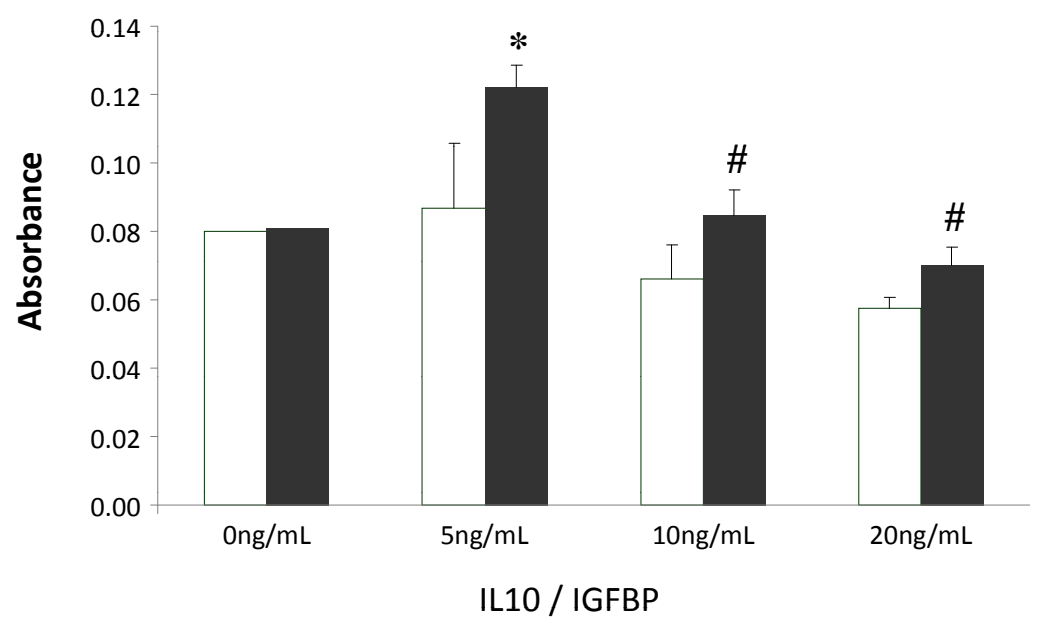

Fig. (4). Effect of IL-10/IGFBP on naïve and CD3 T-cells. Naïve (white with black border) and CD3 (black) T-cells were treated with increasing concentrations of IL-10/IGFBP and proliferation was measured. CD3 T-cells show significant suppression of proliferation as the concentration of IL-10/IGFBP increased from $5 \mathrm{ng} / \mathrm{mL}$ to $20 \mathrm{ng} / \mathrm{mL}$. The proliferation of both naïve and CD3 T-cells was less than control at 10 and $20 \mathrm{ng} / \mathrm{mL}$. Data shown are representative of two independent experiments each done in quadruplicate. Values are means \pm standard errors. There was no significant effect of IL10/IGFBP on naive T-cells, but a there was a statistically significant effect of IL10/IGFBP on CD3 T-cells $(\mathrm{P}<0.05)$ on proliferation $(1$ way ANOVA). *, $\mathrm{P}<.05$ versus no IL10/IGFBP; \#, $\mathrm{P}<.05$ versus 5 ng/mL IL10/IGFBP $($ Bonferonni multiple comparisons).

and therefore this mechanism is not a likely candidate for glioma induction of immunosuppression. Since LDH release is an index of severe cell damage or death, current studies to measure more subtle effects on T-cell viability, such as measures of intracellular ATP levels or of mitochondrial function, need to be done to rule out low-levels of damage induced by GCMs. Second, our results indicate that proliferation suppression is a mechanism of glioma immunosuppression as seen with decreased proliferation of CD3/CD28 T-cells when incubated with increasing concentrations of GCM. This response, though, did not occur specifically with

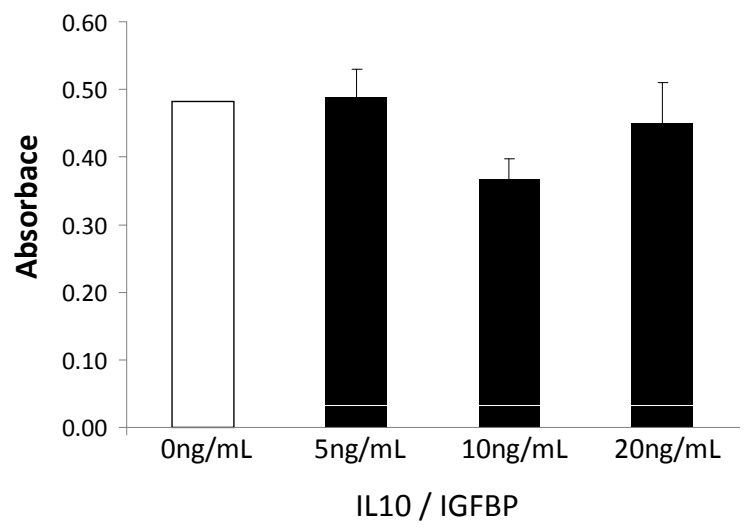

Fig. (5). Effect of IL-10/IGFBP on CD3/CD28 T-cells. CD3/CD28 T-cells were treated with increasing concntrations of IL-10/IGFBP and proliferation was measured. CD3/CD28 T-cells showed a decrease in proliferation as the concentration of IL10/IGFBP increased from $5 \mathrm{ng} / \mathrm{mL}$ to $10 \mathrm{ng} / \mathrm{mL}$ but the proliferation was then increased as the concentration of IL10/IGFBP increased to $20 \mathrm{ng} / \mathrm{mL}$. The proliferation of CD3/CD28 T-cells was less than control. Data shown are representative of two independent experiments each done in quadruplicate. Values are means \pm standard errors. IL10/IGFBP at $10 \mathrm{ng} / \mathrm{mL}$ showed an almost $(\mathrm{P}=$ 0.06 ) statistically significant effect on $\mathrm{CD} 3 / \mathrm{CD} 28 \mathrm{~T}$-cell proliferation compared to control cells and 5 and $20 \mathrm{ng} / \mathrm{mL}$.
aGCM but occurred in nGCM as well. The proliferation inhibition response is therefore not a mechanism that is unique to glioma cells encountering active immunity and could therefore be a base-line glioma immunosuppressive mechanism. The fact that aGCM had an opposite effect on naïve Tcells (increase proliferation) compared to CD3/CD28 T-cells highlights how the immunological status of T-cells not only effects glioma response but also determines how they respond to glioma-secreted cytokines.

The combination of recombinant IL-10 and IGFBP decreased the proliferation of CD3-activated T-cells. In CD3/CD28 activated T-cells, IL-10/IGFBP showed a de-

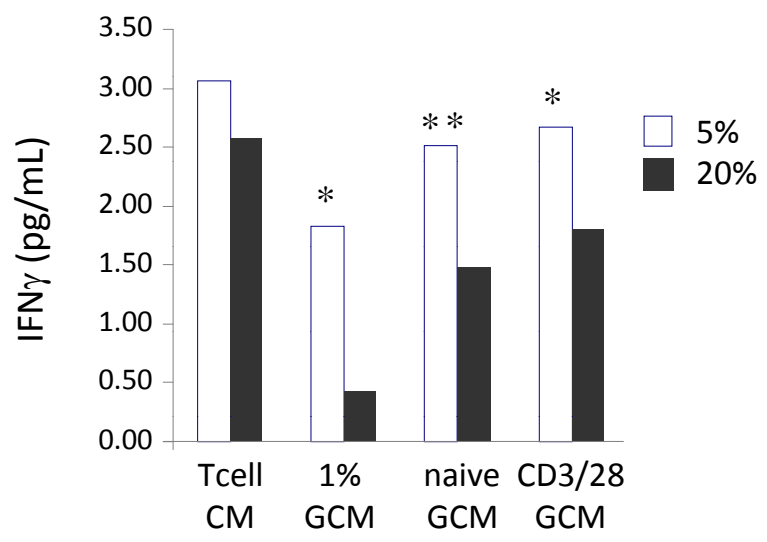

Fig. (6). Effect of GCM on CD3/CD28 T-cell IFN- $\gamma$ release. CD3/CD28 T-cells were treated GCM and assayed for IFN- $\gamma$ release. $\mathrm{CD} 3 / \mathrm{CD} 28$ showed a decrease in IFN- $\gamma$ release when treated with increasing concentrations of all three variations of GCM (1\%GCM, aGCM, and nGCM). Data shown are representative of two independent experiments each done in quadruplicate. Values are means and all standard errors were $<.2 \mathrm{ng} / \mathrm{mL}$. Unpaired t-tests showed statistically significant effects between 5\% versus $20 \%$ with all media tested. *, $\mathrm{P}<0.05$; **, $\mathrm{P}<.005$ versus $20 \%$ media. 
crease as well, but at a concentration of $10 \mathrm{ng} / \mathrm{mL}$ suggesting that this concentration is either optimal for proliferation inhibition or IL-10/IGFBP are not as clearly involved in proliferation of CD3/CD28 activated T-cells as they are in CD-3 T-cells. This combination of cytokines is being further studied for their role in T-cell proliferation.

CD3/CD28 activated T-cells showed a decreased IFN- $\gamma$ release when treated with increasing concentrations of $1 \%$ GCM, activated GCM and naïve GCM. Since IFN- $\gamma$ is mainly secreted by Th1 cells [9] a decrease in IFN- $\gamma$ release suggests a modification of the T-cell subtypes, although it is also possible that the GCM induces expression of antiinflammatory mediators which reduce T-cell IFN- $\gamma$ production (or secretion). These preliminary results suggest that $\mathrm{T}$ cell differentiation may play a role in cytokine-induced immunosuppression of activated T-cells. This response was seen in all three conditions of GCM suggesting that the cytokines responsible for differentiation are secreted constitutively by glioma cells and are not affected by the immunological status of T-cells in the environment. Further studies of T-cell differentiation including flow-cytometry are currently underway.

When recombinant IL-10, TGF- $\beta$ and IGFBP were incubated with T-cells individually, no significant patterns of Tcell proliferation or IFN- $\gamma$ release were noted emphasizing the idea that cytokine-induced immunosuppression requires several cytokines working together. This has to be kept in mind when considering implementation of these mechanisms into treatment and that blocking individual cytokines (ie: TGF- $\beta$ ) is likely less effective than blocking several cytokines together. Currently explored mechanisms of cytokineinduced glioma immunosuppression have been restricted to the immunosuppressive properties of individual cytokines [5]. Suppression of TGF- $\beta$ with antisense [4,8], TGF- $\beta$ kinase inhibitors and other TGF- $\beta$ antagonists were shown to be efficacious in several glioma models improving median survival [10] and reducing tumor volume [11], however the effects were limited. Further elaboration into TGF- $\beta$ 's mechanism regarding synergistic cytokines and the mechanism of decreased cytotoxicity are necessary and could aid in future treatment protocols. In other studies, IL-10 was shown to decrease T-cell proliferation and to down-regulate MHCII on monocytes [5-7]. Our preliminary data showed that IL10 did not significantly suppress T-cell proliferation individually but did in combination with IGFBP. In previous studies we showed that IGFs and IGFBPs were important autocrine growth factors. IGFBP is expressed by many tumors and has been shown to regulate the availability of Insulin Growth Factors (IGF's) [12]. We found that IGFBPs (specifically IGFBP-1) are preferentially expressed in glioma cell lines and are often found in CSF of glioma patients [1215]. IGFBP is currently being considered as a marker for tumor and treatment [12-15]. Other cytokines (PGE2 and VEGF) have also been shown to regulate the immune response [4-8] but also with limited results. These studies are potentially confounded by the glioma's expression of a variety of cytokines that work together to obtain immunosuppression. The blockade of any one cytokine may not be expected to have a significant impact and, as suggested by this study, it is important to first understand the immunosuppressive mechanisms of all collective glioma-secreted cytokines and then to elucidate the combinations of cytokines responsible for these mechanisms. This will increase the effectiveness of treatment by blocking all cytokines involved.

We recently reported encouraging preliminary results of an Immuno-Gene therapy tumor vaccine $[16,17]$ as a treatment for malignant brain tumors. Mice with intracerebral glioma were treated with intratumoral injections of allogeneic fibroblasts genetically modified to secrete IL-2. Compared to control, these mice survived significantly longer. The intratumoral injection caused CD8 T-cell and natural killer cell mediated cytotoxicity in neoplastic cells only, not astrocytes or other non-neoplastic cells [16, 17]. Despite these encouraging results, the mice eventually developed significant tumors, which we attribute to tumor immunosuppression, possibly through the mechanisms elaborated above including suppression of proliferation and T-cell differentiation. With further elaboration of these immunosuppressive mechanisms and cytokines involved, we plan to modify our treatment to include the blockade of newly-found immunosuppressive cytokines and the modification of T-cell differentiation to Th1 and cytotoxic T-cells.

The major mechanisms involved in cytokine-induced immunosuppression described in this study include T-cell proliferation inhibition and T-cell differentiation. On an individual level, the cytokines studied were not enough to mimic the effects seen with GCM but a combination of two (IL-10 and IGFBP) showed similar results to GCM suggesting that $\mathrm{T}$-cell proliferation and differentiation are more likely the product of several cytokines synergistically affecting T-cells. Further studies elaborating immunosuppressive mechanisms of the entire cytokine milieu as well as the identification of cytokine combinations responsible for immunosuppression are currently underway and will hopefully add to our arsenal of information used to treat glioma.

\section{REFERENCES}

[1] Sakaguchi S. Naturally arising CD4+ regulatory T cells for immunologic self-tolerance and negative control of immune responses. Annu Rev Immunol 2004; 22: 531.

[2] Misra N, Bayry J, Lacroix-Desmazes S, et al. Cutting edge: human CD4+CD25+ $\mathrm{T}$ cells restrain the maturation and antigen-presenting function of dendritic cells. J Immunol 2004; 172: 4676.

[3] Taams LS, van Amelsfort JM, Tiemessen MM, et al. Modulation of monocyte/macrophage function by human CD4+CD25+ regulatory T cells. Hum Immunol 2005; 66: 222.

[4] Fakhrai H, Dorigo O, Shawler DL, et al. Eradication of established intracranial rat gliomas by transforming growth factor beta antisense gene therapy. Proc Natl Acad Sci USA 1996; 93: 2909.

[5] Platten M, Wick W, Weller M. Malignant glioma biology: role for TGF-beta in growth, motility, angiogenesis, and immune escape. Microsc Res Tech 2001; 52: 401.

[6] Roszman T, Elliott L, Brooks W. Modulation of T-cell function by gliomas [see comments]. Rev Immunol Today 1991; 12: 370.

[7] Tada M, de Tribolet N. Recent advances in immunobiology of brain tumors. J Neuro Oncol 1993; 17: 261.

[8] Proietti E, Greco G, Garrone B, et al. Importance of cyclophosphamide-induced bystander effect on $\mathrm{T}$ cells for a successful tumor eradication in response to adoptive immunotherapy in mice. J Clin Invest 1998; 101: 429.

[9] Jung HC, Huang MW, Hsieh SL. Enhanced secretion of IFN- $\gamma$ by activated Th1 cells occurs via reverse signaling through TNFrelated activated-induced cytokine. J Immunol 2001; 166: 270-6.

[10] Uhl M, Aulwurm S, Wischhusen J, et al. SD-208, a Novel transforming growth factor B receptor I Kinase inhibitor, inhibits growth and invasiveness and enhances immunogenicity of murine 
and human glioma cells in vitro and in vivo. Cancer Res 2004; 64: 7954.

[11] Platten M, Wild-Bode C, Wick W, et al. N-[3,4Dimethoxycinnamoyl]-Anthranilic acid (Tranilast) inhibits transforming growth factor-B release and reduces migration and invasiveness of human malignant glioma cells. Int J Cancer 2001; 93: 53 .

[12] Zumkeller W, Westphal M. The IGF/IGFBP system in CNS malignancy. J Clinical Pathol 2001; 54: 227-9.

[13] Glick RP, Gettleman R, Patel K, et al. Insulin and insulin-like growth factor I in brain tumors: binding and in vitro effects. Neurosurgery 1989; 24 (6): 791-7.
[14] Glick RP, Unterman TG, Hollis R. Radioimmunoassay of insulinlike growth factors in cyst fluid of central nervous system tumors. J Neurosurgery 1991; 74: 972-8.

[15] Glick RP, Unterman TG, Van der Woude M, Blaydes LZ. Insulin and insulin-like growth factors in central nervous system tumors: Part V: Production of insulin-like growth factors I and II in vitro. J Neurosurgery 1992; 77: 445-50.

[16] Lichtor T, Glick RP. Cytokine immune-gene therapy for treatment of brain tumors. J Neuro Oncol 2003; 65: 247-59.

[17] Lichtor T, Glick RP, Tarlock K, Moffet S, Mouw E, Cohen EP. Application of interleukin-2 secreting syngenec/allogeneic fibroblasts in the treatment of primary and metastatic brain tumors. Cancer Gene Ther 2002; 9: 464-9.

(C) Ksendzovsky et al.; Licensee Bentham Open.

This is an open access article licensed under the terms of the Creative Commons Attribution Non-Commercial License (http://creativecommons.org/ licenses/by-nc/3.0/) which permits unrestricted, non-commercial use, distribution and reproduction in any medium, provided the work is properly cited. 